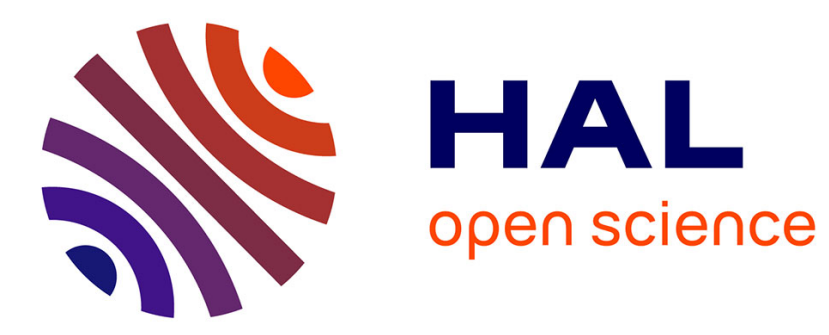

\title{
ELECTRON CORRELATIONS IN PHOTOIONIZATION AND VACANCY DECAY PROCESSES
}

H. Kelly

\section{- To cite this version:}

H. Kelly. ELECTRON CORRELATIONS IN PHOTOIONIZATION AND VACANCY DECAY PROCESSES. Journal de Physique Colloques, 1978, 39 (C4), pp.C4-16-C4-26. 10.1051/jphyscol:1978403 . jpa-00217437

\section{HAL Id: jpa-00217437 https://hal.science/jpa-00217437}

Submitted on 1 Jan 1978

HAL is a multi-disciplinary open access archive for the deposit and dissemination of scientific research documents, whether they are published or not. The documents may come from teaching and research institutions in France or abroad, or from public or private research centers.
L'archive ouverte pluridisciplinaire HAL, est destinée au dépôt et à la diffusion de documents scientifiques de niveau recherche, publiés ou non, émanant des établissements d'enseignement et de recherche français ou étrangers, des laboratoires publics ou privés. 


\title{
ELECTRON CORRELATIONS IN PHOTOIONIZATION AND VACANCY DECAY PROCESSES
}

\author{
H. P. KELLY \\ Department of Physics, University of Virginia, Charlottesville, Virginia 22901, U.S.A.
}

\begin{abstract}
Résumé. - L'influence des effets de corrélation électronique sur les sections efficaces de photoionisation et les taux de désexcitation est discutée dans le cadre de la théorie des perturbations à $N$ corps. Des comparaisons sont effectuées avec d'autres méthodes théoriques telles que la méthode RPAE ou celles introduisant la matrice $R$.
\end{abstract}

\begin{abstract}
The problem of calculating the effects of electron correlations on photoionization cross sections and vacancy decay rates is discussed in the framework of many-body perturbation theory. Comparisons are made with other theoretical methods such as the Random Phase Approximation with Exchange (RPAE) and with the $R$-matrix techniques.
\end{abstract}

1. Introduction. - At the IVth International Conference on Vacuum Ultraviolet Radiation Physics, theoretical atomic photoionization cross sections were discussed and reviewed by Amusia [1] and by Wendin [2]. The discussions centered primarily on the use of the Random Phase Approximation with Exchange (RPAE) first used in atomic physics by Altick and Glassgold [3] who calculated photoionization cross sections for the outer $(n \mathrm{~s})^{2}$ subshells of $\mathrm{Be}, \mathrm{Mg}, \mathrm{Ca}, \mathrm{Sr}$. For an earlier review of photoionization work see Fano and Cooper [4]. RPAE calculations were later carried out on all the rare gases by Amusia, Cherepkov and Chernysheva [5], on $\mathrm{Xe}$ and $\mathrm{Ba}$ by Wendin $[2,6,7]$. These calculations, which generally gave very good agreement with experiment, were limited to closedshell systems. We note that the RPAE includes certain classes of terms in perturbation theory to all orders. These are the most important terms for a dense electron gas [8]. Electron densities in atomic shells are, however, too low to qualify as a dense electron gas in which $r_{s} \leqslant 1[8]$ and it is not clear that this set of terms should be singled out for atoms. In some RPAE papers it is implied or stated that one is dealing with collective effects. However, it has been found that low-order Many Body Perturbation Theory (MBPT) calculations can give good agreement with experiment [9] when the appropriate LS-coupled Hartree-Fock potential is used [5]. Also, MBPT is readily applied to open-shell atoms [10].

Interesting results for open-shell atoms such as $\mathrm{C}, \mathrm{N}, \mathrm{O}$ and $\mathrm{Al}$ have recently been obtained by the $R$-matrix method and are reviewed by Burke [11] and by Le Dourneuf [12]. This article reviews MBPT photoionization calculations which are compared with results obtained by other methods. Vacancy decay processes are also discussed.

2. Many-body perturbation theory. - We consider $N$ identical fermions interacting through two-body potentials $v_{i j}$ in addition to one-body potentials. The Hamiltonian is

$$
\mathscr{H}=\sum_{i=1}^{N} T_{i}+\sum_{i<j}^{N} v_{i j}
$$

where $T_{i}$ is the sum of the kinetic energy operator for the $i$ th particle and all one-body potentials acting on the $i$ th particle. We use atomic units throughout. For atoms of atomic number $Z$,

$$
T_{i}=-\frac{\nabla_{i}^{2}}{2}-\frac{Z}{r_{i}}
$$

and $v_{1 j}=r_{i j}$. An initial approximation is to replace $\mathfrak{J e}$ by

$$
\mathfrak{H}_{0}=\sum_{i=1}^{N}\left(T_{i}+V_{i}\right)
$$

where $\sum v_{i j}$ has been approximated by $\sum V_{i}$. The unperturbed wave function $\Phi_{n}$ for the $n$th state satisfies

$$
\mathscr{H}_{0} \Phi_{n}=E_{n}^{(0)} \Phi_{n},
$$

where $\Phi_{n}$ is a determinant or linear combination of determinants containing $N$ single-particle solutions $\varphi_{i}$ which satisfy

$$
(T+V) \varphi_{i}=\varepsilon_{i} \varphi_{i}
$$


Correlation effects can be included by using perturbation theory with

$$
\mathcal{H}^{\prime}=\sum_{i<j}^{N} v_{i j}-\sum_{i=1}^{N} V_{i} .
$$

We may obtain the solution $\Psi_{n}$ of

$$
\mathcal{H}_{n}=E_{n} \Psi_{n}
$$

by using the many-body perturbation theory of Brueckner [13] and Goldstone [14] according to which

$$
\Psi_{n}=\sum_{L}\left(\frac{1}{E_{n}^{(0)}-\mathscr{H}_{0}} \mathscr{H}^{\prime}\right)^{n} \Phi_{n},
$$

where $\sum_{L}$ indicates that only linked terms are included.

When the system is perturbed by an external electric field, we have the additional perturbation

$$
V_{\text {ex }}=\sum_{i=1}^{N} \mathbf{r}_{i} \cdot \mathbf{E}
$$

and now $\mathcal{H}^{\prime}$ in equation (8) is the sum of the correlation perturbation $\mathcal{H}^{\prime}$ of equation (6) and $V_{\mathrm{ex}}$.

The expression for the photoionization cross section $\sigma_{p}(\omega)$ for the state $\Psi_{p}$, where $\omega$ is the photon energy, is [9]

$$
\sigma(\omega)=(8 \pi \omega / c k)\left|\left\langle\Psi_{k}\left|\sum_{i=1}^{N} \hat{\varepsilon} \cdot \mathbf{r}_{i}\right| \Psi_{p}\right\rangle\right|_{1}^{2}
$$

The energy of the outgoing electron is $k^{2} / 2$ and the final state $\Psi_{k}$ contains a continuum orbital normalized according to

$R_{k}(r) \rightarrow \cos \left[k r+\delta_{l}+(q / k) \ln 2 k r-\frac{1}{2}(l+1) \pi\right] / r$,

as $r \rightarrow \infty$, where $V(r) \rightarrow q / r$. The photon polarization is denoted $\hat{\boldsymbol{\varepsilon}}$.

The many-body matrix element $\left\langle\Psi_{k}\left|\sum \hat{\boldsymbol{\varepsilon}}, \mathbf{r}_{i}\right| \Psi_{p}\right\rangle$ may be expanded in a perturbation series represented by diagrams as shown in figure 1 . The lowest-order diagram, shown in figure $1 a$, is given by $\langle k|z| p\rangle$ where $k$ and $p$ are single-particle states and $\hat{\boldsymbol{\varepsilon}}=\hat{\mathbf{z}}$.

The diagrams are read from bottom to top corresponding to the perturbation proceeding from right to left. The dashed line ending in a heavy dot represents interaction with $V_{\text {ex }}$ (i.e., with $z$ ). The other dashed lines represent Coulomb correlation interactions. Coulomb interactions below the heavy dot represent correlations in the initial state $\Psi_{p}$ and those above the heavy dot represent correlations in the final state $\Psi_{k}$. Figures $1 b, c, d$ represent the diagrams first-order in the correlation correction. The diagram of $1 b$ represents initial state correlations and is given by

$$
\sum_{k^{\prime}} \frac{\left\langle q|z| k^{\prime}\right\rangle\left\langle k k^{\prime}|v| p q\right\rangle}{\varepsilon_{p}+\varepsilon_{q}-\varepsilon_{k}-\varepsilon_{k^{\prime}}},
$$

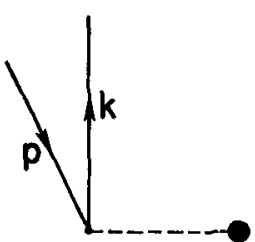

(a)

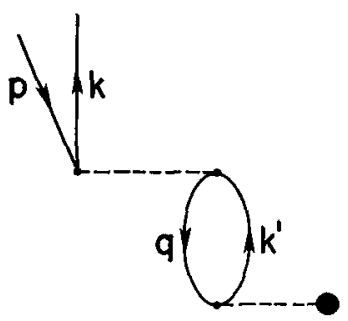

(c)

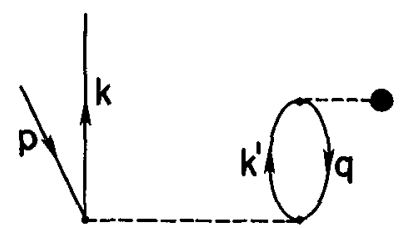

(b)

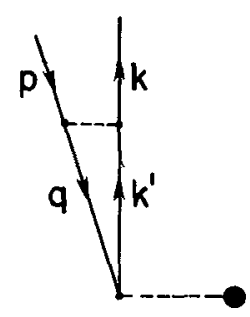

(d)
FIG. 1. - Diagrams contributing to the matrix element

$$
\left\langle\Psi_{k}\left|\sum z_{i}\right| \Psi_{0}\right\rangle
$$

Solid dot indicates matrix element of $z$. Other dashed lines represent Coulomb interaction.

where states $k^{\prime}$ are excited single-particle states. The exchange of $1 b$ should also be included and is obtained by replacing $\left\langle k k^{\prime}|v| p q\right\rangle$ by $-\left\langle k^{\prime} k|v| p q\right\rangle$. Diagrams $1 c$ and $1 d$ represent final state correlation. Diagram $1 c$ is given by

$$
\sum_{k^{\prime}} \frac{\left\langle k q|v| p k^{\prime}\right\rangle\left\langle k^{\prime}|z| q\right\rangle}{\varepsilon_{q}-\varepsilon_{k^{\prime}}+\omega+i \eta},
$$

where the $+\omega$ in the denominator is the photon energy which is present in the initial state and $\eta \rightarrow 0^{+}$. Diagram $1 d$ is the exchange of $1 c$ and is obtained by replacing $\left\langle k q|v| p k^{\prime}\right\rangle$ by $-\left\langle q k|v| p k^{\prime}\right\rangle$ in equation (13).

In equations (12) and (13) sums over continuum states are replaced by integrations, and denominators as in equation (13) are treated according to

$$
\begin{aligned}
& \lim _{\eta \rightarrow 0}\left(\varepsilon_{p}-\varepsilon_{k^{\prime}}+\omega+i \eta\right)^{-1}= \\
& \quad=P\left(\varepsilon_{p}-\varepsilon_{k^{\prime}}+\omega\right)^{-1}-i \pi \delta\left(\varepsilon_{p}-\varepsilon_{k^{\prime}}+\omega\right) .
\end{aligned}
$$

For a discussion of techniques for applying MBPT to atoms, see reference [15].

In figure 2 are shown a large number of second-order correlation diagrams. For a more complete list see reference [16]. Diagrams $2 a$ and $b$ involve creation and annihilation of particle-hole pairs and are the only diagrams of figure 2 included in the RPAE. Diagrams $2 c$ and $d$ involve relaxation effects. Diagram $2 c$, when $r=p$, is part of a geometric series which may be summed to obtain a more accurate ionization energy than is given by the Hartree-Fock description [17]. 
$\left.p\right|_{q} ^{k}$

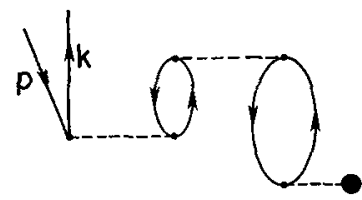

(b)

(a)

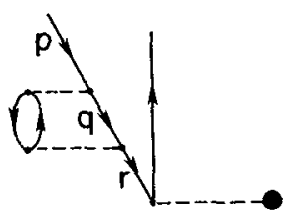

(c)

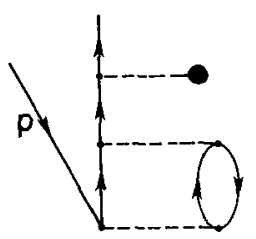

(e)

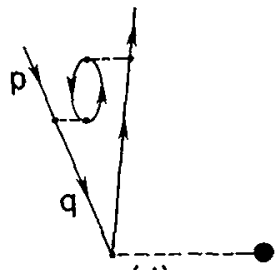

(d)

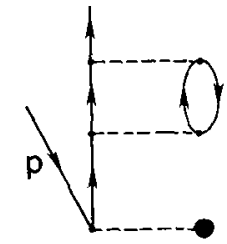

(f)
FIG. 2. - Higher-order diagrams contributing to $\left\langle\Psi_{k}\left|\sum z_{i}\right| \Psi_{0}\right\rangle$. Diagrams $(a)$ and $(d)$ are included in RPAE.

Diagrams $2 e$ and $f$ account for polarization of the atom by the active electron in the initial state and final state respectively.

3. Photoionization cross sections. -3.1 Neutral. ARGON. -- In applying MBPT to $\sigma(\omega)$ for argon [18] we start from a Hartree-Fock ground state $\Phi_{0}$.
The excited Hartree-Fock orbitals were calculated in the Hartree-Fock potential appropriate for the $(3 p)^{5}{ }^{2} \mathrm{P} k{ }^{1} \mathrm{P}$ state. As pointed out by Amusia, Cherepkov and Chernyskeva [5], this potential eliminates the diagrams of figures $1 c, \mathrm{I} d$, and $2 a$ when all the hole states ( $p, q$ and $r$ ) belong to the same subshell. This type potential was also proposed by Ishihara and Poe [19]. In this case, the lowest-order correlation diagram within a given subshell is the initial state correlation diagram of figure $1 \mathrm{~b}$. Calculations by Kelly and Simons [18] are shown in figure 3. The curve labelled HFL is the Hartree-Fock Length curve obtained with the dipole operator $\sum z_{i}$. The curve labelled HFV is the Hartree-Fock Velocity curve obtained using the relation

$$
\left\langle n^{\prime}|\mathbf{r}| n\right\rangle=-\left(E_{n^{\prime}}-E_{n}\right)^{-1}\left\langle n^{\prime}|\nabla| n\right\rangle,
$$

where $H_{n} \Psi_{n}=E_{n} \Psi_{n}$. The experimental results are from Madden, Ederer, and Codling [20] below $37 \mathrm{eV}$ and from Samson [21] above $37 \mathrm{eV}$. The calculation included diagrams $1 b$ and $2 b$ in the (3p) ${ }^{6}$ subshell. The effect of $1 b$ was to bring the length and velocity results into good agreement with each other and with experiment. Diagram $2 b$ had some effect in lowering the calculated curve in the region $30-40 \mathrm{eV}$ to bring it into better agreement with experiment. The window-like resonances above $26 \mathrm{eV}$ were obtained from diagram $1 c$ and $d$ with $p=3 \mathrm{p}, q=3 \mathrm{~s}$, and $k^{\prime}=4 \mathrm{p}, 5 \mathrm{p}$, etc... It was also important to include diagram $2 a$ with $p$, $r=3 \mathrm{p}$ and $q=3 \mathrm{~s}$. In diagrams $1 c$ and $2 a$ there are vanishing denominators for $\omega$ such that

$$
\varepsilon_{3 \mathrm{~s}}-\varepsilon_{n p}+\omega=0 \text {. }
$$

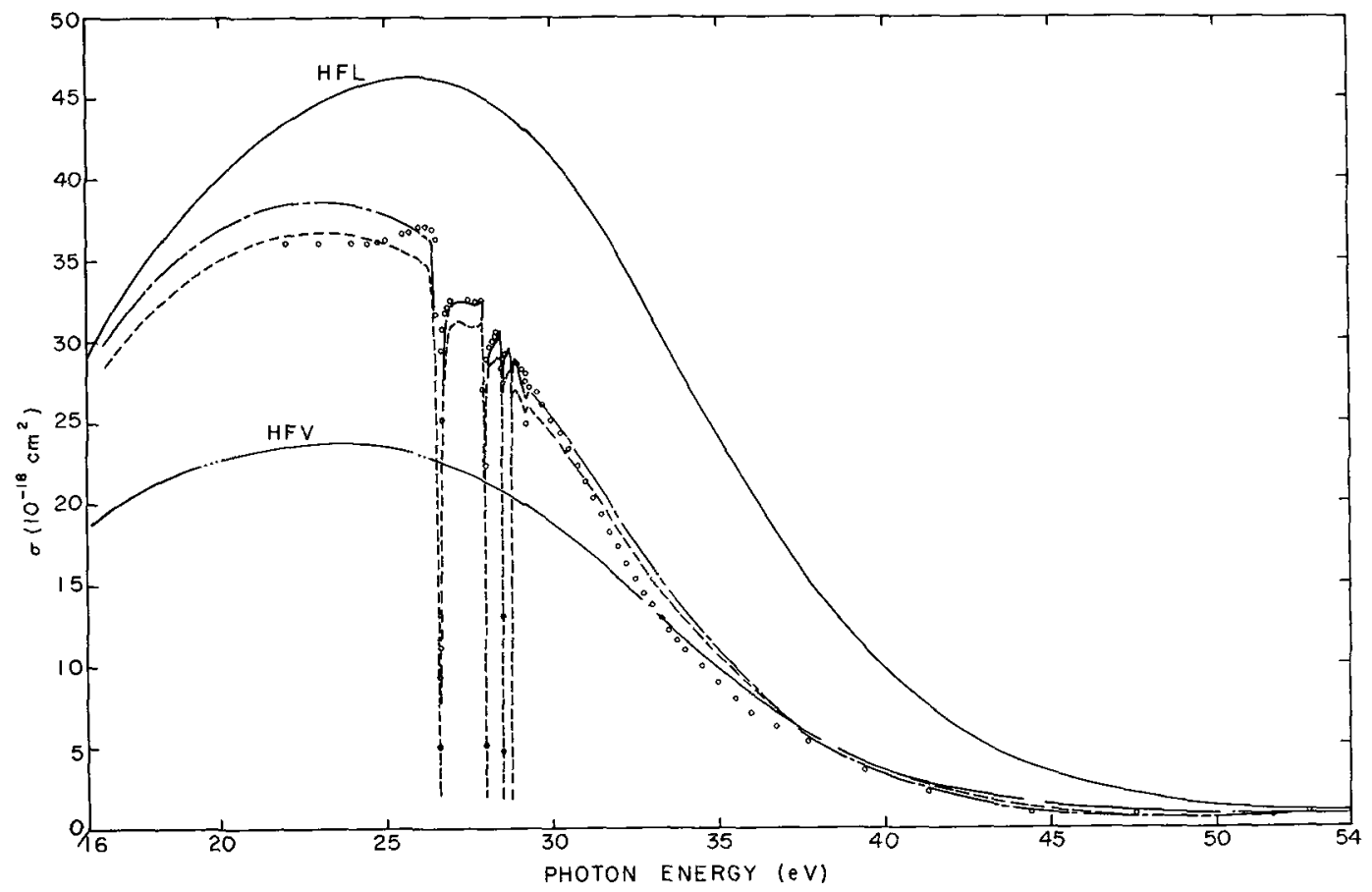

FIG. 3. - Cross section for photoionization by argon. Calculations from reference [18]. HFL and HFV are Hartree-Fock length and velocity cross sections. Dot-dashed (dashed) line is calculated length (velocity) cross section including higher-order terms. Circles are experimental data from reference [20] below $37 \mathrm{eV}$ and from reference [21] above $37 \mathrm{eV}$. Only the lowest $3 \mathrm{~s} \rightarrow n$ p resonances are shown. 
Including higher-order diagrams, an exact geometric sum may be made which shifts these denominators to

$$
\varepsilon_{3 \mathrm{~s}}-\varepsilon_{n \mathrm{p}}+\Delta+i \Gamma_{n} / 2
$$

where $\Gamma_{n}$ is the width of the $3 \mathrm{~s} 3 \mathrm{p}^{6} n \mathrm{p}^{1} \mathrm{P}$ state and $A$ is the usual resonance shift. In summary, excellent agreement is obtained by a low-order perturbation calculation if one uses Hartree-Fock continuum states appropriate for the $(3 \mathrm{p})^{5}{ }^{2} \mathrm{P} k \mathrm{~d}{ }^{1} \mathrm{P}$ state. Other calculations for argon are shown in figure 4 and compared

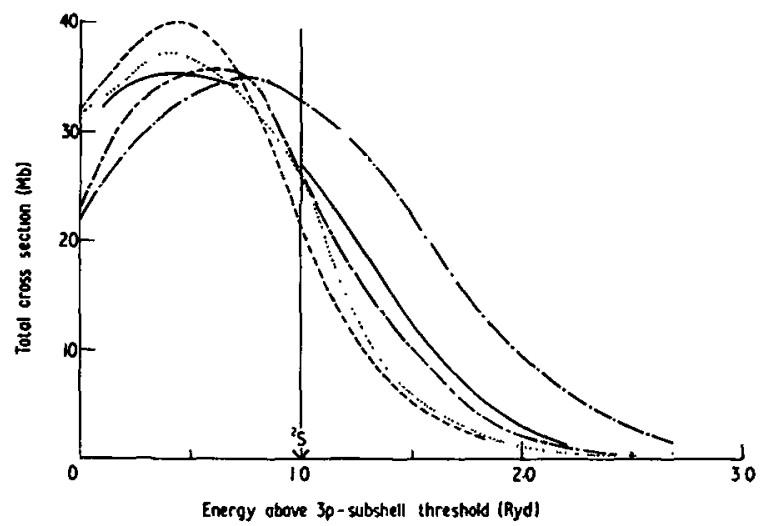

FIG. 4. - Photoionization cross section of argon. The full curve is the $R$-matrix length calculation of Burke and Taylor [22]. Dotted curve is experimental results of Samson [25]. Broken curve is RPAE calculation [5]. Dash-dot curve is $K$-matrix calculation [24] and long dashed curve is an SRPAE calculation [23].

with experiment. The solid line is a length calculation by Burke and Taylor [22] using $R$-matrix theory. The calculations by Lin [23] used a simplified Random Phase Approximation (SRPAE) approach. The dashdot curve is the $K$-matrix calculation of Starace [24], and the dotted curve shown is the experimental results by Samson [25]. The RPAE calculations tend to be too large near threshold, and this effect is much greater in some other cases such as $\mathrm{Ba}$ to be discussed later.

Swanson and Armstrong [26] have successfully used the multiconfiguration Hartree-Fock method to include ground state correlation effects for the rare gases. They have recently improved these results by also using a multiconfiguration potential in the final state (Swanson and Armstrong [27]). Chang [28] has also obtained good results for neon and argon with a type of coupled differential equation form of the RPAE based on a formalism of Chang and Fano [29].

The 3 s cross section in argon is quite interesting in that it is changed qualitatively when $3 \mathrm{~s}-3 \mathrm{p}$ correlations are included by diagrams $1 b, c$, and $d$ with $p=3 \mathrm{~s}$ and $q=3 \mathrm{p}$. Calculations of this effect have been made by Amusia, Ivanov, Cherepkov, and Chernysheva [30] using the RPAE, by Lin using the SRPAE [23], by Burke and Taylor [23] using the $R$-matrix, and by Pindzola and Kelly [31] in a loworder MBPT calculation. The dash-dot curve is the Hartree-Fock calculations of Cooper and Manson [32].
The results of these calculations are compared with the experimental results of Marr et al. [33] in figure 5. The crosses are experimental values by Samson and Gardner [34].

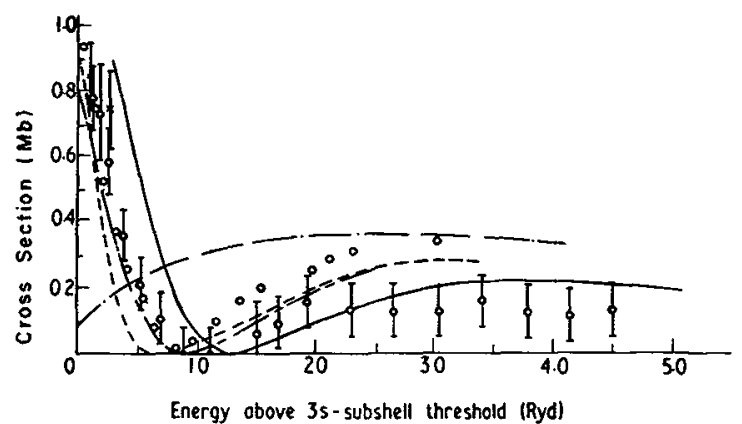

FIG. 5. - Argon 3s-subshell cross section. Full curve is $R$-matrix calculation [22], crosses are experimental values of Samson and Gardner [34], circles with error bars are experimental data of Marr [33], dash-dot curve is Hartree-Fock calculation [32], broken curve is RPAE calculation [30], and long dashed curve is SRPAE calculation [23]. Open circles are MBPT calculation [31].

3.2 Photoionization of $\mathrm{Zn}$. - Calculations of $\sigma(\omega)$ for Zn by Fliflet and Kelly [35] give another illustration of the very great effect of correlations on a subshell with a small $\sigma(\omega)$ when a neighbouring subshell has a large $\sigma(\omega)$. This effect was just illustrated for the $3 \mathrm{~s}$ subshell of argon. The configuration for $\mathrm{Zn}$ is $(3 \mathrm{~d})^{10}(4 \mathrm{~s})^{2}{ }^{1} \mathrm{~S}$. In this case $\sigma_{4 s}(\omega)$ is small

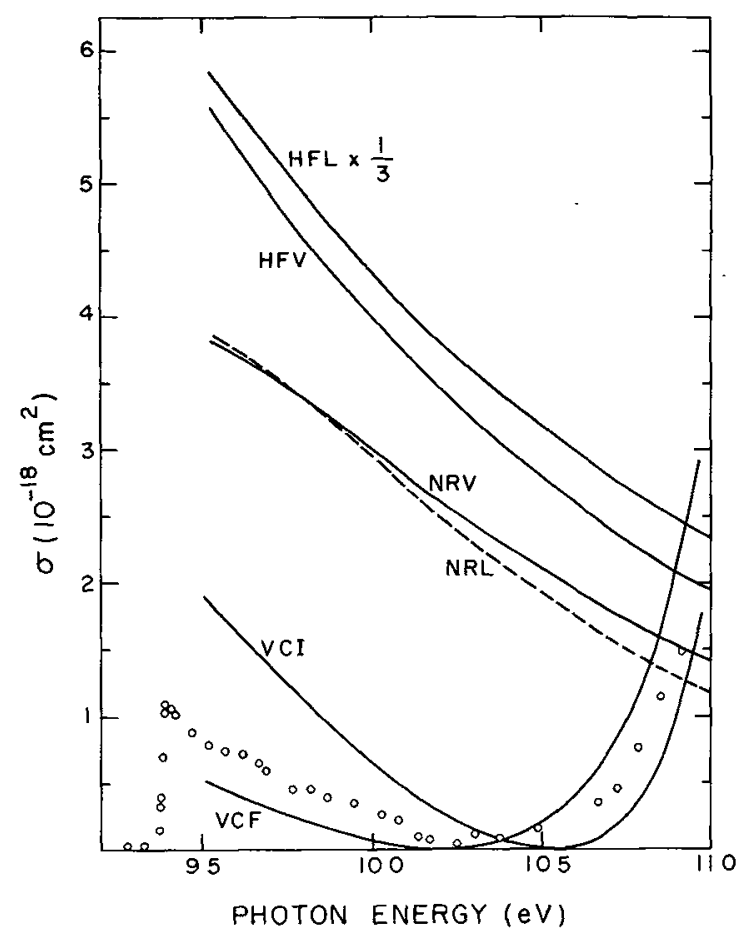

FIG. 6. - Calculated cross section for photoionization of the $4 \mathrm{~s}$ subshell of ZnI near threshold [35]. Curves HFL and HFV are Hartree-Fock length and velocity cross sections using frozen-core orbitals. Curves NRL and NRV are the non-resonant correlated curves. Curves VCF and VCI are correlated velocity cross sections with frozen-core and ionic core orbitals respectively. 
relative to $\sigma_{3 \mathrm{~d}}(\omega)$. Important correlation effects were found from diagrams $1 b$ with $p=4 \mathrm{~s}$ and $q=3 \mathrm{~d}$ or $4 \mathrm{~s}$ and from $1 c$ and $d$ with $p=4 \mathrm{~s}$ and $q=3 \mathrm{~d}$. Selected higher-order diagrams were also included. Calculated results are shown in figure 6 and compared with the experimental values of Marr and Austin [36]. The curves labelled NRV and NRL are Non-Resonant Velocity and Length curves respectively. They were calculated without including the $3 \mathrm{~d} \rightarrow 4 \mathrm{p}, 5 \mathrm{p}$, etc... resonance excitations in diagrams such as $1 c$ and $d$ and similar higher-order diagrams. The curves VCI and VCF are final velocity results including resonance excitations for the $4 \mathrm{~s} \rightarrow k \mathrm{p}$ excitations calculated with an ionic core and a frozen core potential respectively. In both cases, the length and velocity results agreed closely. The very dramatic effects of correlations are seen in the fact that the Hartree-Fock Velocity (HFV) and Hartree-Fock Length (HFL) curves differ from experiment by factors of 6 and 18 respectively near threshold. Figure 7 shows a comparison between the calculated and measured $\sigma_{4 \mathrm{~s}}(\omega)$ in the region of the $3 d \rightarrow 4 p$ resonances. Due to spinorbit splitting, there are separate resonances due to the ${ }^{1} \mathrm{P}_{1},{ }^{3} \mathrm{P}_{1}$, and ${ }^{3} \mathrm{D}_{1}$ levels of the $(3 \mathrm{~d})^{9}(4 \mathrm{~s})^{2} 4 \mathrm{p}$ configuration. The window between the ${ }^{1} \mathrm{P}_{1}$ and ${ }^{3} \mathrm{D}_{1}$ resonances was obtained in the calculations when the appropriate diagrams were included to account for interaction between resonances.

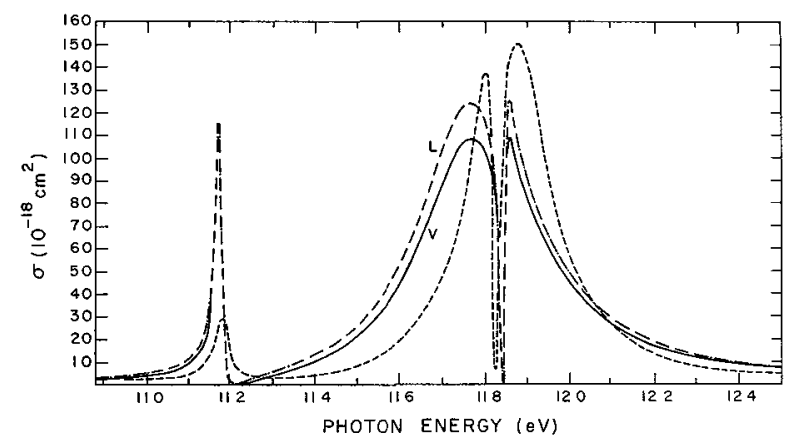

FIG. 7. - Zinc photoionization cross section in the $3 d^{9} 4 s^{2} 4 p$ resonance region. Solid line, correlated velocity cross section. Dashdot line, correlated length cross section [35]. Dashed line, experimental data from Marr and Austin [36].

3.3 Photolonization of barium. - The cross section for the $(4 \mathrm{~d})^{10}$ subshell of barium is a good example of the use of low-order MBPT. Calculations [37] were carried out using Hartree-Fock continuum states appropriate to the $(4 \mathrm{~d}){ }^{9}{ }^{2} \mathrm{D} \mathrm{kp}{ }^{1} \mathrm{P}$ and $(4 \mathrm{~d})^{9}{ }^{2} \mathrm{D} k \mathrm{f}^{1} \mathrm{P}$ states. The Hartree-Fock length and velocity curves were found to bracket the experimental curve of Connerade and Mansfield [38] which was normalized to give an oscillator strength of 10 and is shown in figure 8 . Including the Ground State Correlation diagram (GSC) of figure $1 b$ brought the length and velocity curves into close agreement. The calculated dashed curve shown in figure 8 is an

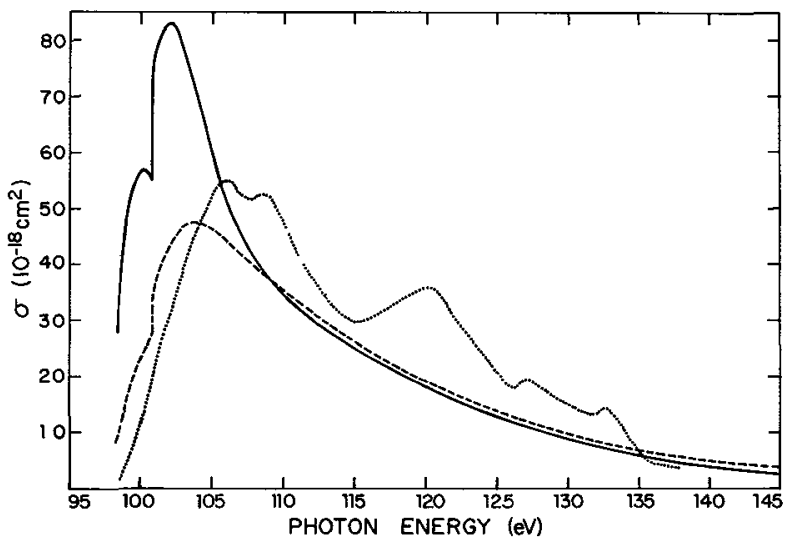

FIG. 8. - Calculated [37] and experimental results for $\sigma_{4 \mathrm{~d}}$ of BaI : -, average of length and velocity from RPAE results including splittıng between $4 d^{9}{ }^{2} D_{5 / 2}$ and ${ }^{2} D_{3 / 2}$ cores $; \ldots$. experimental data of Connerade and Mansfield [38] normalized to an oscillator strength of 10. ....-, average of length and velocity ground state correlation results including spin-orbit splitting of cores.

average of the length and velocity GSC results. The effect of splitting between the $4 \mathrm{~d}^{9}{ }^{2} \mathrm{D}_{5 / 2}$ and ${ }^{2} \mathrm{D}_{3 / 2}$ cores was obtained by assigning the cross section according to statistical weights. The RPAE curve calculated by Fliflet et al. [37] is much too large near threshold and is seen to give worse agreement with experiment than their low-order MBPT curve. The experimental $\sigma(\omega)$ measured by Rabe et al. [39] is also in good agreement with the MBPT curve of figure 8. Much of the original interest in the (4d) ${ }^{10}$ subshell of Ba was due to a calculation by Wendin [7] which predicted a collective resonance near $120 \mathrm{eV}$ but the low-order MBPT calculation is in better agreement with experiment. We note, however, that the RPAE calculations for the (4d) ${ }^{10}$ subshell of Xe by Amusia et al. [5] are in good agreement with experiment.

3.4 Photolonization of Cadmium (4d) ${ }^{10}(5 \mathrm{~s})^{2}$. This cross section was calculated [40] by MBPT for the (4d) ${ }^{10}$ subshell using Hartree-Fock excited states appropriate to (4d) ${ }^{9}{ }^{2} \mathrm{D} k \mathrm{p}{ }^{1} \mathrm{P}$ and $(4 \mathrm{~d})^{9}{ }^{2} \mathrm{D} k \mathrm{f}^{1} \mathrm{P}$. Length and velocity curves were calculated, and ground state correlations were included by the diagram of figure $1 b$ which brought the length and velocity curves into close agreement. In order to test

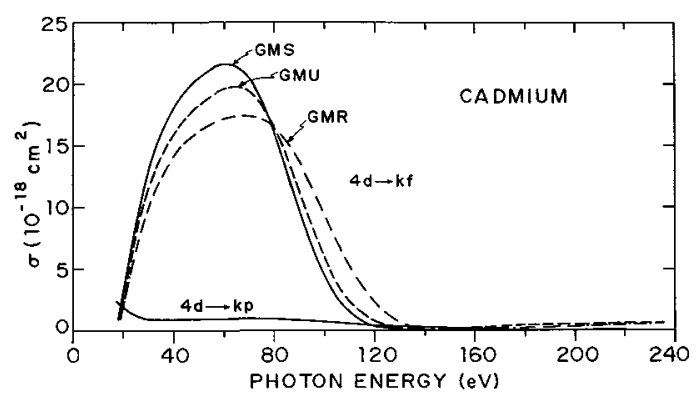

Fig. 9. - Photoionization cross sections for the (4d) ${ }^{10}$ subshell of CdI calculated by Carter and Kelly [40]. All curves contain geometric mean of length and velocity results. MGU uses unrelaxed orbitals, GMU uses relaxed orbitals, and GMS contains the secondorder RPAE diagrams. 
for higher-order corrections, the RPAE diagram of figure $2 b$ was included and the resulting curve is shown in figure 9 labelled GMS. This curve is a Geometric Mean (GM) of the length and velocity curves (see Hansen [41]) which are already close. The curve labelled GMU is the Geometric Mean of the Unrelaxed length and velocity results obtained from diagrams $1 a$ and $b$. That is, only ground state correlations are included beyond the Hartree-Fock treatment.

In order to include the effects of relaxation, which are accounted for by diagrams $2 b$ and $c$, the experimental removal energy was used in all calculations. Excited states were calculated in the field of the relaxed ion $(4 \mathrm{~d})^{9}{ }^{2} \mathrm{D}(5 \mathrm{~s})^{2}$. Physically, one expects that when the departing electron velocity is low the ion will have time to relax and so the outgoing electron moves in the field of the relaxed ion. At higher energies, the atom will not have time to relax and so a frozen core approach is more appropriate. Calculations in the field of the relaxed ion including ground state correlations are shown in figure 9 by the curve labelled GMR. Again the geometric mean between length and velocity was taken. It is clear from figure 9 that the effects of higher-order RPAE diagrams and of relaxation are in opposite directions and roughly cancel. One formal objection to using orbitals calculated in a relaxed potential is that they will not always be orthogonal to occupied orbitals of the same symmetry. In $\mathrm{Cd}$ for $4 \mathrm{~d} \rightarrow k \mathrm{f}$ excitations there is no problem because there are no occupied forbitals.

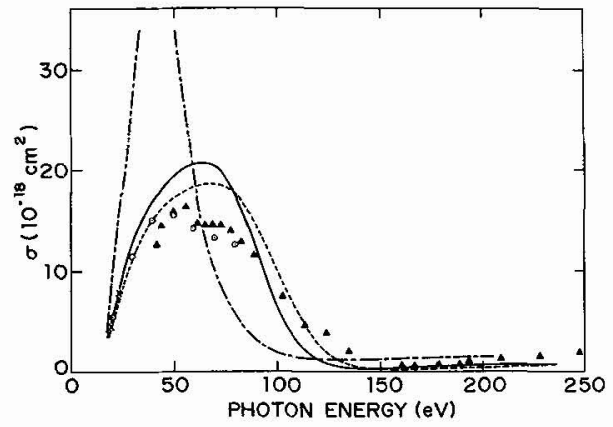

FIG. 10. - Comparison of calculated and measured cross sections for CdI. —, MBPT frozen core calculation [40]. . . . . : MBPT relaxed core calculation [40]. - . - . - Hartree Fock Slater calculation by McGuire [45]. Experimental values : $\Delta$, Codling, et al. [42],

$\odot$, Cairns et al. [43], $\times$, Marr and Austin [44].

In figure 10 the calculated results (including both $4 \mathrm{~d} \rightarrow k \mathrm{f}$ and $4 \mathrm{~d} \rightarrow k \mathrm{p}$ ) are compared with experimental results (triangles) by Codling et al. [42], by Cairns et al. [43] shown by circles, and by Marr and Austin [44] shown by crosses. The solid curve includes ground state correlations with geometric mean between length and velocity and excited states calculated in the frozen core potential. The dashed curve is the same but with relaxed excited states. Also shown for comparison is the calculated cross section in the Hartree-Fock-Slater approximation by McGuire [45].
The importance of the correct treatment of exchange is very clear. Also, the relaxed calculation appears to be in a little better agreement with experiment in this case than the frozen core calculation.

Recently Amusia, Ivanov and Chernysheva [46] have carried our RPAE calculations which used relaxed excited states. These results for $\mathrm{Xe}, \mathrm{Cs}$, and $\mathrm{Ba}$ agreed better with experiment than the usual RPAE results.

3.5 Photoionization of OPEN-Shell atoms. Recently there have been some detailed calculations of $\sigma(\omega)$ for open-shell systems of low $Z$. Calculations on $\mathrm{Al}, \mathrm{B}, \mathrm{C}, \mathrm{N}$ and 0 have been carried out using the $R$-matrix approach of Burke and Taylor [22] and are reviewed by Le Dourneuf [12]. Calculations for $\mathrm{Si}^{+}$[47] and for C [10] have used MBPT. Recent calculations for $\mathrm{Cl}$ have used the RPAE (Starace and Armstrong [48], Cherepkov and Chernysheva [49]), MBPT [50], and the $R$-matrix [51].

The calculations for $\mathrm{Al} 3 \mathrm{~s}^{2} 3 \mathrm{p}{ }^{2} \mathrm{P}$ by Le Dourneuf, Vo Ky Lan, Burke and Taylor [52] using the $R$-matrix approach are very impressive. They have obtained excellent agreement with the experimental curve of Kohl and Parkinson [53] which has a threshold value of $65 \pm 7 \mathrm{Mb}\left(1 \mathrm{Mb}=10^{-18} \mathrm{~cm}^{2}\right)$. This is to be compared with previous one-electron threshold values of $29 \mathrm{Mb}$ [54], $20 \mathrm{Mb}$ [55], and $22 \mathrm{Mb}$ [56]. They [52] have found very large correlation effects in the ${ }^{2} \mathrm{D}$ channel due to configuration mixing of $3 s \mathrm{~s}^{2}{ }^{2} \mathrm{D}$ with $3 s^{2} n d^{2} \mathrm{D}$ and $3 \mathrm{~s}^{2} k \mathrm{~d}{ }^{2} \mathrm{D}$.

Calculations using MBPT for C [10] may be compared with the $R$-matrix calculations by Taylor and Burke [57] who also calculated $\sigma(\omega)$ for oxygen. Both calculations are in reasonable agreement (within $20 \%$ as to the magnitude of the cross section. The MBPT results are shown in figure 11. Both calculations also show resonance structure, and figure 12 shows reasonable agreement of the MBPT cross section in the region of the $2 s 2 p{ }^{4} \mathrm{P} n \mathrm{p}$ series with experimental

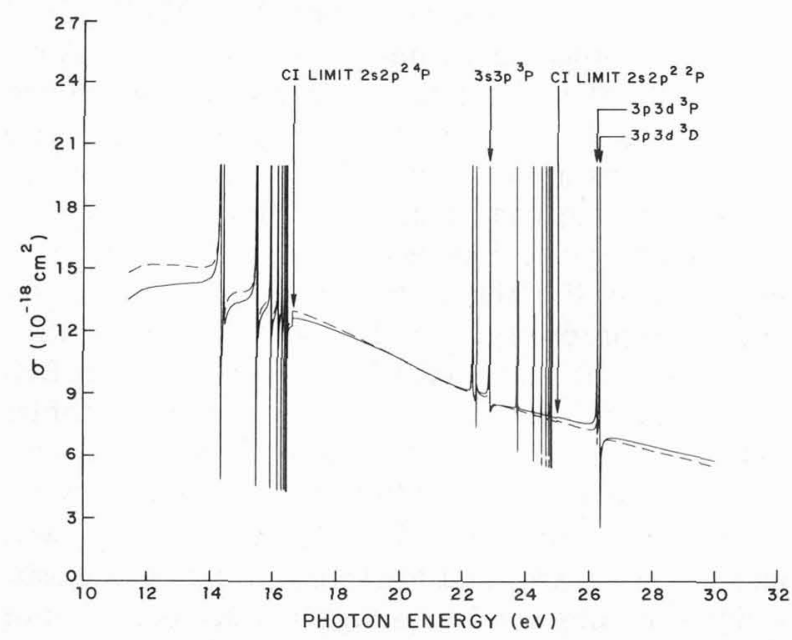

Fig. 11. - Total ${ }^{3} \mathrm{P}$ carbon photoionization cross section calculated by Carter and Kelly [16]. Solid line is length form. Dashed line is velocity form. Resonance peaks truncated at $20 \mathrm{Mb}$. 


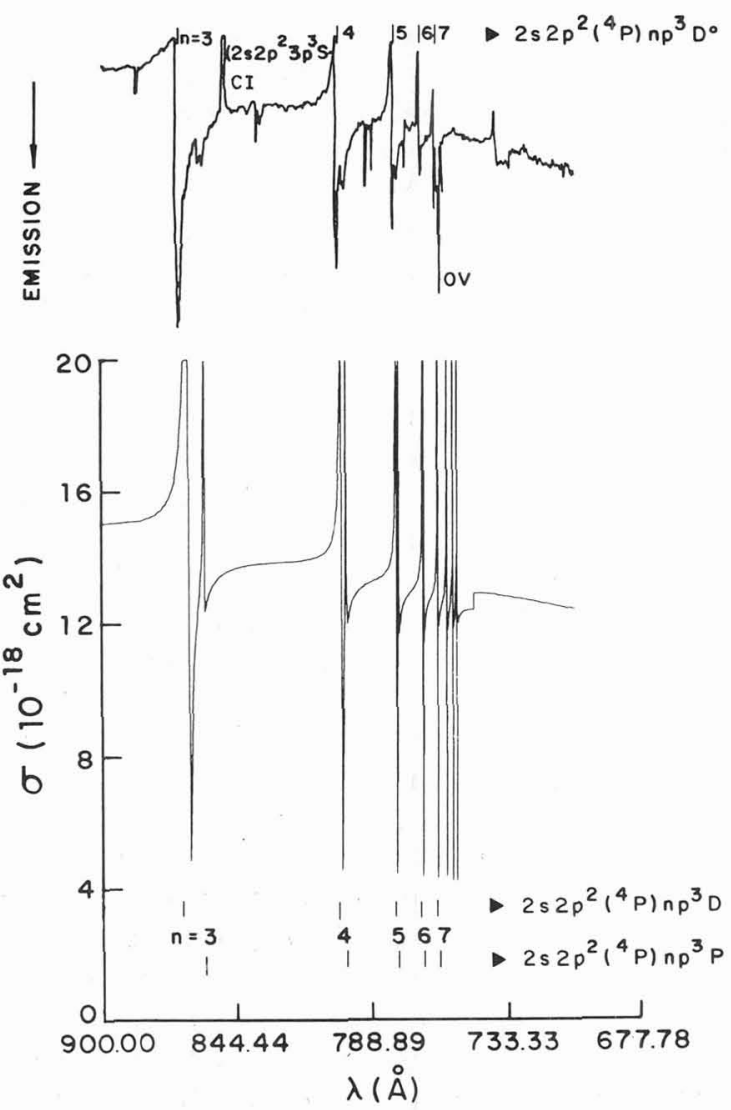

FIG. 12. - Detail of velocity form curve of figure 11 in the resonance region of the $2 \mathrm{~s} 2 \mathrm{p}^{2}\left({ }^{4} \mathrm{P}\right) n \mathrm{p}$ series. The unnormalized densitometer trace is from reference [58].

data by Esteva et al. [58]. The $R$-matrix curve is also in good agreement with experiment [58]. However, as pointed out by Taylor and Burke [57], there is disagreement between the resonance widths from the two calculations. For the $2 \mathrm{~s} 2 \mathrm{p}^{2}{ }^{3} \mathrm{P} 3 \mathrm{p}{ }^{3} \mathrm{D}$ resonance the width is $3.72 \times 10^{-2} \mathrm{eV}(R$-matrix $)$ and $8.77 \times 10^{-3} \mathrm{eV}$ (MBPT). A close examination of the experimental data indicates a value approximately half-way between the two calculations. It should be noted the MBPT result is a lowest-order calculation for the width and is not necessarily of high accuracy. One could, of course, proceed to higher orders to improve the result. The $R$-matrix result is in effect a higher-order calculation by the fact of coupling different configurations. Further calculations by both methods appear to be desirable. For the $2 \mathrm{~s} 2 \mathrm{p}^{2}{ }^{4} \mathrm{P} 3 \mathrm{p}{ }^{3} \mathrm{P}$ resonance, the differences are even greater, $5.22 \times 10^{-2} \mathrm{eV}(R$-matrix $)$ and $5.71 \times 10^{-3} \mathrm{eV}(\mathrm{MBPT})$. In this case the MBPT result included the $k \mathrm{~d}$ final orbit but not the contribution from $k$ s and so is too small. However, it is not clear that this can account fully for the discrepancy.

The autoionization series $2 \mathrm{~s} 2 \mathrm{p}^{2}{ }^{2} \mathrm{D},{ }^{2} \mathrm{~S} n \mathrm{p}$ were included by Taylor and Burke but not by Carter and Kelly since this involves a higher-order perturbation treatment.

The extensive calculations for the nitrogen atom by Le Dourneuf et al. [59] are of considerable interest.
There has been considerable theoretical interest in $\sigma(\omega)$ for the $(3 p)^{5}{ }^{2} \mathrm{P}$ ground state of the chlorine atom since the interesting open-shell RPAE calculation of Starace and Armstrong [48] based on the openshell RPAE formulation by Armstrong [60]. This work found the very surprising result that the correlation effects were very small and that the Hartree-Fock results were little changed by including correlations by the open-shell RPAE method. This is surprising since the correlation effects in argon with only one more $3 p$ electron are considerable. Very recent results now dispute the Starace and Armstrong [48] calculation shown in figure 13 by closed circles. Results by Cherep-

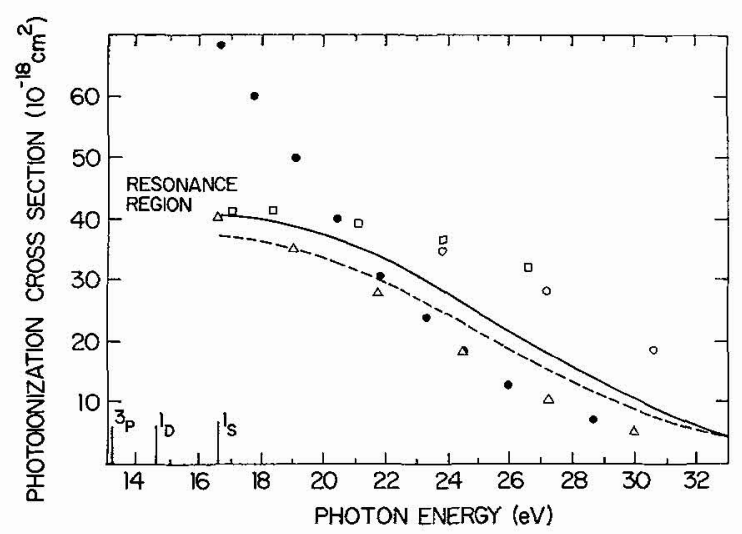

FIG. 13. - Calculated photoionization cross sections for the $(3 p)^{5}$ subshell of ground state chlorine atoms. $3 \mathrm{~s}$ excitations not included. -, MBPT length calculation [50]. - - ., MBPT velocity calculation [50]. RPAE calculation by Starace and Armstrong [48] A. RPAE calculation by Cherepkov and Chernysheva [49]. $\square$, close coupling calculation by Conneely, et al. [61]. O, $R$-matrix calculation by Lamoureux and Combet-Farnoux [51].

kov and Chernysheva [49] which used an openshell RPAE formalism are shown by triangles. $R$-matrix calculations by Lamoureux and CombetFarnoux [51] are represented by open circles. The MBPT results [50] are shown by the solid line (length) and dashed line (velocity). Previous close-coupling results [61] are shown by crosses. In all cases the results near threshold are much lower than those of Starace and Armstrong, and the general shape of $\sigma(\omega)$ is reasonably close to that obtained for argon. The MBPT calculations shown do not include the $3 \mathrm{~s}$ subshell cross section or correlation effects with $3 \mathrm{~s}$ electrons although such results will soon be presented [50]. In the MBPT calculation there are large final state correlation effects involving coupling between the $3 \mathrm{p}^{4}{ }^{3} \mathrm{P} k \mathrm{~d}, 3 \mathrm{p}^{4}{ }^{1} \mathrm{D} k \mathrm{~d}$, and $3 \mathrm{p}^{4}{ }^{1} \mathrm{~S} k \mathrm{~d}$ channels and these were included to all orders by solving a set of coupled integral equations. These couplings include the diagrams like figure $1 c$ and $d$ and figure $2 a$ and corresponding higher-order diagrams, all of which can be included in the closed-shell case by the appropriate choice of potential. These diagrams for the chlorine case are very important in lowering $\sigma(\omega)$ from the Hartree-Fock value near 
threshold. It appears that these couplings involving the different $3 \mathrm{p}^{4}$ multiplets of $\mathrm{Cl}^{+}$were not included in the calculation by Starace and Armstrong [48]. Since the $R$-matrix calculations in argon [22] were higher than experiment after the peak in $\sigma(\omega)$, it may be that the $R$-matrix calculations for $\mathrm{Cl}$ are high in the range $20-35 \mathrm{eV}$.

3.6 Double Photolonization. - Recent experimental work on multiple electron photoionization of rare gases by Schmidt et al. [62], Wight and Van der Wiel [63], Samson and Haddad [64], Wuilleumier and Krause [65], Lightner et al. [66], Cairns et al. [43] and Carlson [67] has shown that these processes can contribute a substantial percentage of the total ionization cross section. So far only MBPT has produced results for atoms larger than $\mathrm{He}$. Calculations for the double photoionization cross section $\sigma^{2+}(\omega)$ of neon have been made by Chang and Poe $[68,69]$ and by Carter and Kelly [70], for argon [71], and for carbon [72]. In all these calculations the lowestorder diagrams of MBPT involving the dipole interaction and one Coulomb correlation interaction were evaluated for the dipole many-body matrix element. This is a much more complicated process than single photoionization because, among other things, an infinite number of combinations of partial waves contribute. Tabile 1 shows the various contri-

\section{TABLE I}

\section{Dipole transitions}

\begin{tabular}{|c|c|c|c|}
\hline & $\begin{array}{c}\text { Contribution }\left({ }^{\circ}\right) \\
(0 / 0)\end{array}$ \\
\hline - & \multicolumn{2}{|c|}{ State $\left({ }^{a}\right)$} & - \\
\hline \multirow[t]{4}{*}{$3 s^{2} 3 p^{61} S$} & $3 \mathrm{~s}^{2} 3 \mathrm{p}^{4}\left({ }^{3} \mathrm{P}, !^{1} \mathrm{D}\right.$ & S) $k \mathrm{~s} k \mathrm{p}\left(\mathrm{L}^{\prime}\right){ }^{1} \mathrm{P}$ & 6.7 \\
\hline & & $k \mathrm{p} k \mathrm{~d}$ & 63.7 \\
\hline & & $k \mathrm{~s} k \mathrm{f}$ & 1.8 \\
\hline & & $k \mathrm{~d} k \mathrm{f}$ & 6.8 \\
\hline \multirow[t]{3}{*}{$3 s^{2} 3 p^{6}{ }^{1} S$} & $3 s 3 p^{5}\left({ }^{3} P,{ }^{1} P\right)$ & $k^{\prime} \mathrm{p} k \mathrm{p}\left(\mathrm{L}^{\prime}\right){ }^{1} \mathrm{P}$ & 9.9 \\
\hline & & $k^{\prime} \mathrm{d} k \mathrm{~d}$ & 1.6 \\
\hline & & $k \mathrm{~s} k \mathrm{~d}$ & 9.5 \\
\hline
\end{tabular}

$\left(^{a}\right)$ Notation $\left(\mathrm{L}^{\prime}\right)$ indicates that all allowed terms of the $k^{\prime} k$ electron pair are to be included which are consistent with the final state coupling.

(b) Average of length and velocity curves at $89.3 \mathrm{eV}$

butions to $\sigma^{2+}(\omega)$ for argon calculated by Carter and Kelly [71] at $89.8 \mathrm{eV}$, approximately the maximum of $\sigma^{2+}(\omega)$. Although the $(3 \mathrm{p})^{2} \rightarrow k \mathrm{p} k \mathrm{~d}$ transition is largest, the other contributions cannot be ignored. It is particularly interesting to note the significant contribution from $3 \mathrm{~s} 3 \mathrm{p} \rightarrow k k^{\prime}$ transitions since $\sigma_{3 \mathrm{~s}}(\omega)$ is itself so small. The total $\sigma^{2+}$ for argon is shown in figure 14. Both length (L) and velocity (V) curves are shown for the lowest order calculations and also for the calculation including a limited number of higherorder diagrams. The calculations are a little lower than experiment, but the omitted partial waves could increase $\sigma^{2+}$ by 10 or $15 \%$. Similar results have been

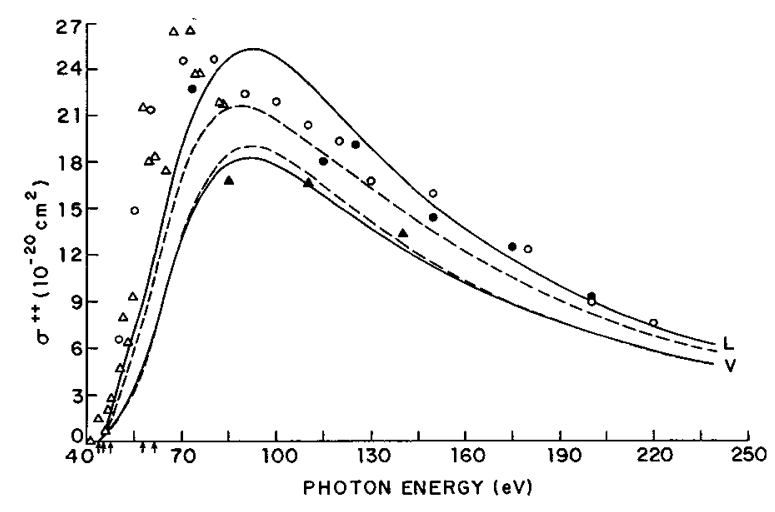

FIG. 14. - Double photoionization cross section $\sigma^{2+}(\omega)$ for neutral argon. Full curves are the lowest-order results for dipole-length $(L)$ and dipole-velocity (V) matrix elements calculated by Carter and Kelly [71]. Broken curves include some second-order correlations. Experimental data points are from Schmidt et al. [62], full circles, Wight and Van der Wiel [63], open circles ; Samson and Hadden [64], open triangles, full triangles Carlson [67]. Arrows mark, respectively, experimental excitation thresholds for $3 s^{2} 3 p^{4}\left({ }^{3} \mathrm{P},{ }^{1} \mathrm{D},{ }^{1} \mathrm{~S}\right)$ and 3s $3 p^{5}\left({ }^{3} \mathbf{P},{ }^{1} \mathbf{P}\right)$ core configurations.

found for $\mathrm{Ne}$ [70] except that the agreenent with experiment near threshold is worse. This has been interpreted as due to the fact that one of the outgoing electrons sees the nucleus screened by $N-2$ electrons rather than $N-1$. The results are in disagreement with the previous $\sigma^{2+}$ calculations for neon [69] which included only $(2 \mathrm{p})^{2} \rightarrow k \mathrm{p} k \mathrm{~d}$ in the dipole velocity form and obtained a curve a little larger than experiment.

4. Auger processes. - In calculating the manybody energy of a system with an inner-shell vacancy, it may be shown that the Auger rate is proportional to the imaginary part of the energy when only coulomb interactions are included and denominators $D$ are replaced by $D+i \eta$ where $\eta \rightarrow 0$ [73]. The real part of the energy contributes to the calculation of the removal energy. Using MBPT, the correlation energy of neon with a ls vacancy was calculated to be 0.0298 a.u. relative to the correlation energy of neutral neon [73]. After estimating relativistic effects, the difference in energy between $\mathrm{Ne}^{+}\left(1 \mathrm{~s}^{-1}\right)$ and $\mathrm{Ne}$ is $870.53 \mathrm{eV}$ [74] as compared with the experimental value $870.2 \mathrm{eV}$ [75]. Similar calculations for the energies of all the K-LL Auger lines of Ne gave results [74] in good agreement with experiment [76].

For the Auger intensities, one may either calculate the imaginary parts of energy diagrams or else calculate Auger matrix elements obtained by separating energy diagrams into two parts where denominators are treated according to $-i \pi \delta(D)$. Diagrams for Auger matrix elements when there is a $1 \mathrm{~s}$ vacancy are shown in figure 15. The diagrams are equivalent to configuration mixing by Rayleigh-Schrödinger perturbation theory. These diagrams were evaluated for all five LS intensities and gave the results shown in table II. The experimental results are from Mehlhorn 


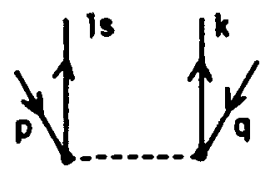

(a)

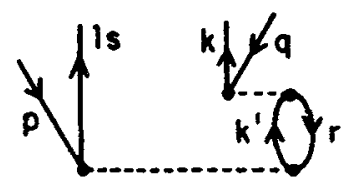

(d)

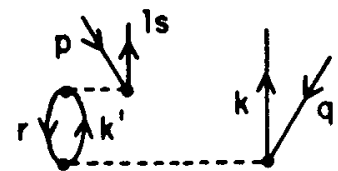

(g)

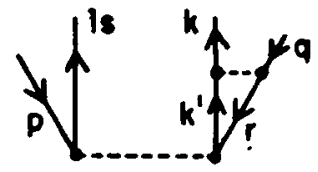

(b)

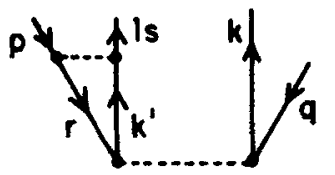

(e)

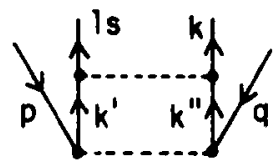

(h)

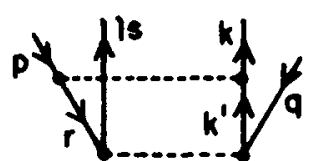

(c)

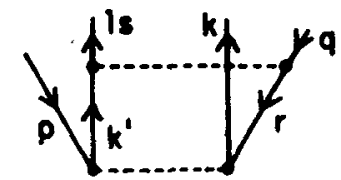

(f)

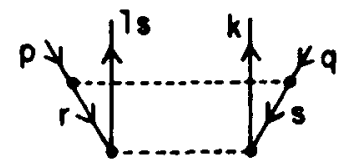

(i)

FIG. 15. - Diagrams contributing to the Auger-rate matrix element for a ls vacancy. $(a)$ lowest order; $(b)$, $(c)$, $(d)$ and $(i)$ are equivalent to configuration mixing in the final state; $(e)-(h)$ represent configuration mixing in initial state.

TABLE II

Auger rates for $\mathrm{Ne}\left(1 \mathrm{~s}^{-1}\right)\left(10^{-3}\right.$ a.u. $)$

\begin{tabular}{ccll} 
Transition & $\mathrm{HF}\left({ }^{a}\right)$ & \multicolumn{1}{c}{$\operatorname{Corr}\left({ }^{b}\right)$} & \multicolumn{1}{c}{ Expt. $\left({ }^{(}\right)$} \\
- & - & - & - \\
$1 \mathrm{~s}-2 \mathrm{~s} 2 \mathrm{~s}\left({ }^{1} \mathrm{~S}\right)$ & 0.951 & $0.488\left(0.701\left(^{d}\right)\right)$ & $0.54 \pm 0.11$ \\
$2 \mathrm{~s}-2 \mathrm{~s} 2 \mathrm{p}\left({ }^{1} \mathbf{P}\right)$ & 2.034 & 1.396 & $1.48 \pm 0.29$ \\
$1 \mathrm{~s}-2 \mathrm{~s} 2 \mathrm{p}\left({ }^{3} \mathrm{P}\right)$ & 0.789 & 0.502 & $0.54 \pm 0.11$ \\
$1 \mathrm{~s}-2 \mathrm{p} 2 \mathrm{p}\left({ }^{1} \mathrm{~S}\right)$ & 0.456 & $0.864\left(0.707\left({ }^{d}\right)\right)$ & $0.85 \pm 0.17$ \\
$1 \mathrm{~s}-2 \mathrm{p} \mathrm{2p}\left({ }^{1} \mathrm{D}\right)$ & 5.685 & 5.198 & $5.05 \pm 0.102$ \\
Total & 9.914 & 8.448 & $8.45 \pm 0.78$
\end{tabular}

$\left({ }^{a}\right)$ Hartree-Fock or lowest order result [74].

(b) Including correlation diagrams of figure 15 [74].

(c) Experimental values from Mehlhorn et al. [77] normalized by the total rate measured by Gelius et al. [78].

$\left({ }^{d}\right)$ Correlations only from mixing of $(1 s)^{2}(2 s)^{2}(2 p)^{4}{ }^{1} S$ and $(1 \mathrm{~s})^{2}(2 \mathrm{p})^{6}{ }^{1} \mathrm{~S}$.

et al. [77] renormalized according to the experiment of Gelius et al. [78].

Consider the $\mathrm{KL}_{1} \mathrm{~L}_{1}$ intensity or Auger rate to $(1 \mathrm{~s})^{2}(2 \mathrm{p})^{6}{ }^{1} \mathrm{~S}$. In figure 15 , diagram (i) with $p=q=2 \mathrm{~s}$ and $r=\mathrm{s}=2 \mathrm{p}$ corresponds to configuration mixing of the final states of $\mathrm{Ne}^{++}(1 \mathrm{~s})^{2}(2 \mathrm{p})^{6}{ }^{1} \mathrm{~S}$ and $(1 s)^{2}(2 s)^{2}(2 p)^{4}{ }^{1} S$ and is important. However, diagrams $(b),(c)$, and $(d)$ with $p, q=2 \mathrm{~s}$ and $r=2 \mathrm{p}$ are equally important. They correspond to configuration mixing of the final state $(1 s)^{2}(2 p)^{6} k s^{2} S$ with states $(1 s)^{2}(2 s)(2 p)^{5} k p^{2} S$. It is seen from table II that the correlation effects can be appreciable, especially for the weaker transitions.

For $\operatorname{Be}\left(1 \mathrm{~s}^{-1}\right)$, or $1 \mathrm{~s} 2 \mathrm{~s}^{2}{ }^{2} \mathrm{~S}$, the most important configuration mixing now occurs in the initial state and involves mixtures of $1 \mathrm{~s} 2 \mathrm{p}^{2}{ }^{2} \mathrm{~S}$ with $1 \mathrm{~s} 2 \mathrm{~s}^{2}{ }^{2} \mathrm{~S}$, as indicated by diagram (h) with $p, q=2 \mathrm{~s}$ and $k^{\prime}$, $k^{\prime \prime}=2 \mathrm{p}$. This increases the lowest-order Hartree-Fock value from $1.81 \times 10^{-3}$ a.u. to $3.42 \times 10^{-3}$ a.u. [79]. As one goes from $\mathrm{Be}$ to $\mathrm{Ne}$, the K-LL rate steadily decreases as the important correlations change from initial state configuration mixing to final state configuration mixing.

5. Radiative transitions. - Most calculations of radiative rates for inner-shell vacancies have neglected correlations, which can be very important in these processes. A review of correlation calculations for such transitions including use of MBPT has been given recently [80]. In addition to studies of one-electron transitions, MBPT may be readily used to calculate transition rates in which two or more electrons change orbitals. A very simple example is Be $1 \mathrm{~s} 2 \mathrm{~s}^{2}{ }^{2} \mathrm{~S}$ state which cannot undergo decay via electric dipole transitions to the $1 \mathrm{~s}^{2} 2 \mathrm{~s}^{2} \mathrm{~S}$ state. However, there is a non-zero electric dipole transition rate to the $1 \mathrm{~s}^{2} n \mathrm{p}{ }^{2} \mathrm{P}$ states when correlated wave functions are used. The largest effect is due to the significant admixture of $1 \mathrm{~s} 2 \mathrm{p}^{2}{ }^{2} \mathrm{~S}$ which can make an electric dipole transition to $1 s^{2} 2 p{ }^{2} \mathrm{P}$. When all first-order correlation perturbations are included for both initial and final states, the radiative rate is $7.34 \times 10^{-3}$ a.u. as compared with $10.21 \times 10^{-3}$ a.u. when only $2 \mathrm{~s}-2 \mathrm{p}^{2}$ mixing in the initial state is included [79].

The two-electron-one-photon transition rate for $\mathrm{Fe}^{++}(2 \mathrm{~s})^{2}(2 \mathrm{p})^{6}(3 \mathrm{p})^{6}(3 \mathrm{~d})^{10}(4 \mathrm{~s})^{2}$ has been calculated in the electron dipole approximation by MBPT [81] and 
compared with the experimental result by Wolfi et al. [82]. The ratio of the one-electron-one-photon rate to the two-electron-one-photon rate was calculated as 5630 (length) and 5860 (velocity) compared with $4000 \pm 3000$ measured by Wolfli et al. [82] and improved recently to $3950 \pm 500$ [83]. Although a large part of the MBPT calculation could be attributed to the effect of monorthogonality of $2 s$ and $1 \mathrm{~s}$ orbitals of the initial and final states, there was still a significant contribution from other correlation effects, especially for the length value. Using only the monorthogonality effect, a value of 1870 was calculated by Gavrila and Hansen [84] and approximately 2600 by Åberg et al. [85]. Recent calculations of two-electron-onephoton transitions have been calculated for the $(1 \mathrm{~s})^{-2}$ state of neon, $(2 s)^{-2}$ and $(2 p)^{-2}$ states in argon, and $(4 \mathrm{~d})^{-2}$ in Xe by Amusia, Lee, and Zinoviev [86] using MBPT.

6. Concluding remarks. - In preceding sections it has been shown that electron correlation effects may often be calculated successfully by at least several different methods. The MBPT and RPAE methods have been used successfully on some rather large closed-shell atoms as well as smaller atoms. The $R$-matrix methods have been successfully applied to the largest number of open-shell atoms. MBPT calculations have been carried out on several small open-shell atoms. Both MBPT and $R$-matrix methods have calculated resonance structure. It is possible that the $R$-matrix method is also preferable in those cases where there is strong coupling with a few configurations as in $\mathrm{Al}$. The $R$-matrix method may be most effective in calculating resonance structure. In cases where there are important correlations involving virtual transitions to continuum states such as for chlorine, argon, barium, xenon, etc..., one might prefer to use MBPT or the RPAE. So far, only MBPT has been used to calculate double photoionization cross sections for atoms larger than helium. All of these methods have advantages, and it is expected that further improvements will be made on each of them in the near future.

Acknowledgments. - I wish to thank the National Science Foundation for financial support and Dr. S. L. Carter for helpful discussions.

\section{References}

[1] Amusia, M. Ya, Proceedings of the IV International Conference on Vacuum Ultraviolet Radiation Physics (eds. E. E. Koch, R. Haensel, ad C. Kunz, Pergamon, Vieweg) 1974, 205.

[2] WENDIN, G., ibid., 225.

[3] Altick, P. L. and Glassgold, A. E., Phys. Rev. 133A (1964) 632.

[4] Fano, U. and Cooper, J. W., Rev. Mod. Phys, 40 (1968) 441.

[5] Amusia, M. Ya, Cherepkov, N. A. and Chernysheva, L. V., Sov. Phys. JETP 33 (1971) 90.

[6] Wendin, G., J. Phys. B 5 (1972) 110.

[7] Wendin, G., Phys. Lett. 46A (1973) 119.

[8] Brueckner, K. A., The Many-Body Problem (Wiley, New York), 1959, 175

[9] KelLy, H. P., in Photoionization and Other Probes of ManyElectron Interactions (ed. F. J. Wuilleumier, Plenum, New York), 1976, 83.

[10] Carter, S. L. and Kelly, H. P., Phys. Rev. A 13 (1976) 1388.

[11] Burke, P. G., J. Physique Colloq. 39 (1978) C4-27.

[12] Le Dourneuf, M., Prog. Rep. X ICPEAC, to be published.

[13] Brueckner, K. A., Phys. Rev. 97 (1955) 1353.

[14] Goldstone, J., Proc. R. Soc. A 239 (1957) 267.

[15] Kelly, H. P., Adv. Chem. Phys. 14 (1969) 129.

[16] Chang, J. J. and Kelly, H. P., Phys. Rev. 12 (1975) 92.

[17] Kelly, H. P., Phys. Rev. A 1 (1970) 274.

[18] Kelly, H. P. and Simons, R. L., Phys. Rev. Lett. 30 (1973) 529.

[19] Ishimara, T. and PoE, R. T., Phys. Rev. A 6 (1972) 111.

[20] Madden, R. P., Ederer, D. L. and Codling, K., Phys. Rev. 177 (1969) 136.

[21] Samson, J. A. R., Adv. At. Mol. Phys. 2 (1966) 177.

[22] Burke, P. G. and Taylor, K. T., J. Phys. B 8 (1975) 2620.

[23] Lin, C. D., Phys. Rev. A 9 (1974) 181.

[24] Starace, A. F., Phys. Rev. A 2 (1970) 118.

[25] Samson, J. A. R., J. Opt. Soc. Am. 55 (1965) 935.

[26] Swanson, J. R. and Armstrong, Jr., L., Phys. Rev. A 15 (1977) 661.

[27] Swanson, J. R. and Armstrong, Jr., L., to be published.

[28] Chang, T. N., Phys. Rev. A 15 (1977) 2392.

[29] Chang, T. N. and Fano, U., Phys. Rev. A 13 (1976) 263, 282.
[30] Amusia, M. Ya, Ivanov, V. K., Cherephov, N. A. and CherNYSHEVA, L. V., Phys. Lett. 40A (1972) 361.

[31] Pindzola, M. S. and Kelly, H. P., Phys. Rev. A 12 (1975) 1419.

[32] Cooper, J. W. and Manson, S. T., Phys. Rev. 177 (1969) 157.

[33] MarR, G. V., unpublished data.

[34] Samson, J. A. R. and Gardner, J. L., Phys. Rev. Lett. 33 (1974) 671 .

[35] Fliflet, A. W. and Kelly, H. P., Phys. Rev. A 10 (1974) 508.

[36] Marr, G. V. and Austin, J. M., J. Phys. B 2 (1972) 168.

[37] Fliflet, A. W., Chase, R. L. and Kelly, H. P., J. Phys. B 7 (1974) L443.

[38] Connerade, J. P. and Mansfield, M. W. D., Proc. R. Soc. A 341 (1974) 267.

[39] RABE, P., RADLER, K. and WolFF, H. W., Vaccum Ultraviolet Radiation Physics (eds. E. E. Koch, R. Haensel and C. Kung, Pergamon, Vieweg), 1974, 247.

[40] Carter, S. L. and Kelly, H. P., to be published.

[41] Hansen, A. E., Mol. Phys. 13 (1967) 425.

[42] Brint, P. R., West, J. B., Codling, K. and Hanley, J. R., to be published.

[43] Cairns, R. B., Harrison, H. and Schren, R. I., J. Chem. Phys. 51 (1969) 5440.

[44] Marr, G. V. and Austin, J. M., Proc. R. Soc. A 310 (1969) 137.

[45] MCGuire, E. J., Phys. Rev. 175 (1968) 20.

[46] Amusia, M Ya, Ivanov, V. K. and Chermysheva, L. V., Phys. Lett. 59A (1976) 191.

[47] Daum, G. R. and Kelly, H. P., Phys. Rev. A 13 (1976) 715.

[48] Starace, A. F. and Armstrong, Jr., L., Phys. Rev. A 13 (1976) 1850 .

[49] Cherepkov, N. A. and Chernysheva, L. V., Phys. Lett. 60A (1977) 103

[50] Brown, E. R., Carter, S. L. and Kelly, H. P., to be published.

[51] Lamoureux, M. and Combet-Farnoux, F., Abstracts of Vth VUV Conference, Montpellier, France (1977) 22.

[52] Le Dourneuf, M., Vo Ky Lan, Burke, P. G. and Taylr, K. T., J. Phys. B 8 (1975) 2640. 
[53] KohL, J. L. and PARKinson, W. H., Astrophys. J. 184 (1973) 641.

[54] Vainstein, L. A. and Norman, G. E., Opt. Spectrosc. 8 (1960) 79.

[55] Peach, G., Mon. Not. R. Astron. Soc. 124 (1962) 371.

[56] Travis, L. D. and Matsushima, S., Astrophys. J. 154 (1968) 689.

[57] Taylor, K. T. and Burke, P. G., J. Phys. B 9 (1976) L353.

[58] Esteva, J. M., Mehlman-Balloffet, G. and Romand, J., J. Quant. Spectrosc. Radiat. Transfer 12 (1972) 1291.

[59] Le Dourneuf, M., Vo Ky Lan and Hibbert, A., J. Phys. $B 9$ (1976) L-359.

[60] Armstrong, Jr., L., J. Phys. B 7 (1974) 2320.

[61] Conneely, M. J., Smith, K. and Lipsky, L., J. Phys. B 3 (1970) 493.

[62] Schmidt, V., Sandner, N., Kuntzemuller, H., Dhez, P., Wuilleumier, F. and Kallne, E., Phys. Rev. A 13 (1976) 1748

[63] Wight, G. R. and VAN Der Wiel, M. J., J. Phys. B 9 (1976) 1319.

[64] Samson, J. A. R. and Haddad, G. N., Phys. Rev. Lett. 33 (1974) 875 .

[65] Wuilleumier, F. and Krause, M. O., Phys. Rev. A 10 (1974) 242.

[66] Lightner, G. S., Van Brunt, R. J. and Whitehead, W. D., Phys. Rev. A 4 (1971) 602.

[67] Carlson, T. A., Phys. Rev. 156 (1967) 142.

[68] Chang, T. N., Ishihara, T. and Poe, R. T., Phys. Rev. Lett. 27 (1971) 838
[69] Chang, T. N. and Poe, R. T., Phys. Rev. A 12 (1975) 1432.

[70] Carter, S. L. and Kelly, H. P., Phys. Rev. A 16 (1977) 1525.

[7I] Carter, S. L. and Kelly, H. P., J. Phys. B 9 (1976) L565.

[72] Carter, S. L. and Kelly, H. P., J. Phys. B 9 (1976) 1887.

[73] Chase, R. L., Kelly, H. P. and Kohler, Phys. Rev. A 3 (1971) 1550.

[74] Kelly, H. P., Phys. Rev. A 11 (1975) 556.

[75] SibgBahn, K. et al., ESCA Applied to Free Molecules (NorthHolland, Amsterdam), 1969.

[76] Korber, H. and Mehlhorn, W., T. Phys. 191 (1966) 217.

[77] Mehlhorn, W., Stalherm, D. and Verbeek, H., Z. Naturforsch. A 23 (1968) 287.

[78] Gelius, U., Svenson, S., Siegbahn, H., Basilier, E., Faxalv, A. and Siegrahn, K., Chem. Phys. Lett. 28 (1974) 1.

[79] Kelly, H. P., Phys. Rev. A 9 (1974) 1582.

180] Kelly, H. P, in Atomic Inner-Shell Processes I (ed. B. Crasemann, Academic, New York), 1975, 331.

[81] Kelly, H. P., Phys. Rev. Lett. 37 (1976) 386.

[82] Wolfli, W., Stoller, Ch., Bonani, G., Suter, M. and StockLI, M., Phys. Rev. Lett. 35 (1975) 656.

[83] Stoller, Ch., Wolfli, W., Bonani, G., Stòckli, M. and Suter, M., Phys. Rev. A 15 (1977) 990.

[84] Gavrila, M. and Hansen, J. E., Phys. Lett. 58A (1976) 158.

[85] Åberg, T., Jamison, K. A. and Richard, P., Phys. Rev. Lett. 37 (1976) 63.

[86] Amusia, M. Ya, Yee, I. S. and Zinoviev, A. N., Phys. Lett. 60A (1977) 300 . 